University of New Hampshire

University of New Hampshire Scholars' Repository

Physics Scholarship

Physics

2006

\title{
Thermal Expansion and Magnetostriction of the Ising Antiferromagnet TbNi2Ge2
}

\author{
G M. Schmiedeshoff \\ Occidental College \\ Shawna M. Hollen \\ S L. Budko \\ lowa State University \\ P C. Canfield \\ lowa State University
}

University of New Hampshire - Main Campus

Follow this and additional works at: https://scholars.unh.edu/physics_facpub

Part of the Physics Commons

\section{Recommended Citation}

G. M. Schmiedeshoff, S. M. Hollen, S. L. Bud'ko, and P. C. Canfield, 'Thermal expansion and Magnetostriction of the Ising Antiferromagnet TbNi2Ge2', in 24th International Conference on Low Temperature Physics, 2006, vol. 850, no. 1, pp. 1297-1298. http://dx.doi.org/10.1063/1.2355182

This Article is brought to you for free and open access by the Physics at University of New Hampshire Scholars' Repository. It has been accepted for inclusion in Physics Scholarship by an authorized administrator of University of New Hampshire Scholars' Repository. For more information, please contact Scholarly.Communication@unh.edu. 


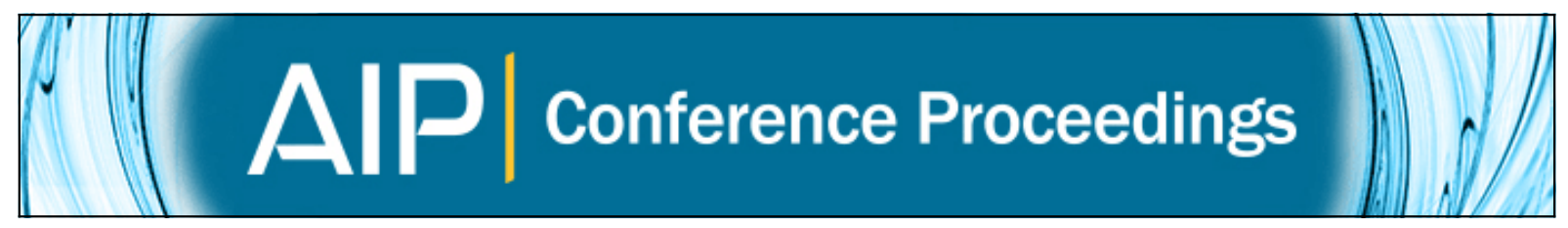

\section{Thermal Expansion and Magnetostriction of the Ising Antiferromagnet TbNi2Ge2}

G. M. Schmiedeshoff, S. M. Hollen, S. L. Bud'ko, and P. C. Canfield

Citation: AIP Conference Proceedings 850, 1297 (2006); doi: 10.1063/1.2355182

View online: http://dx.doi.org/10.1063/1.2355182

View Table of Contents: http://scitation.aip.org/content/aip/proceeding/aipcp/850?ver=pdfcov

Published by the AIP Publishing

Articles you may be interested in

A comparative study of magnetic behaviors in TbNi2, TbMn2 and TbNi2Mn

J. Appl. Phys. 115, 17E135 (2014); 10.1063/1.4869440

Negative thermal expansion and spontaneous magnetostriction of $\mathrm{Tb} 2 \mathrm{Fe} 16.5 \mathrm{Cr} 0.5$ compound J. Appl. Phys. 97, 116102 (2005); 10.1063/1.1921334

Magnetic phase transitions in (Tb,Y)Mn $2 \mathrm{M} 2(\mathrm{M}=\mathrm{Ge}$ and $\mathrm{Si})$ systems

J. Appl. Phys. 93, 8185 (2003); 10.1063/1.1541652

Magnetostriction and angular dependence of ferromagnetic resonance linewidth in Tb-doped $\mathrm{Ni} 0.8 \mathrm{Fe} 0.2$ thin films

J. Appl. Phys. 91, 8659 (2002); 10.1063/1.1452708

First-order antiferromagnetic ordering and metamagnetic transition in ( $\mathrm{TbPd} 3$ ) $8 \mathrm{~T}$ ( $\mathrm{T}=\mathrm{Ga}$ and $\mathrm{Ge})$

J. Appl. Phys. 87, 5146 (2000); 10.1063/1.373277 


\title{
Thermal Expansion and Magnetostriction of the Ising Antiferromagnet $\mathrm{TbNi}_{2} \mathrm{Ge}_{2}$
}

\author{
G.M. Schmiedeshoff*, S.M. Hollen*, S.L. Bud'ko", and P.C. Canfield \\ *Department of Physics, Occidental College, Los Angeles, CA 90041, USA \\ "Ames Laboratory and Department of Physics, Iowa State University, Ames, IA 50011, USA
}

\begin{abstract}
We have measured the linear thermal expansion and magnetostriction of the Ising antiferromagnet $\mathrm{TbNi}_{2} \mathrm{Ge}_{2}$ along its c-axis from room temperature to $2 \mathrm{~K}$ and in magnetic fields to $14 \mathrm{~T}$. We find a magnetic phase diagram that agrees with earlier work and estimate aspects of its uniaxial pressure dependence. We also find a new high field feature near $10 \mathrm{~T}$ which may signal the onset of an additional field-induced phase.
\end{abstract}

Keywords: Thermal Expansion, Magnetostriction, Antiferromagnetism.

65.40.De, 75.50.Ee, 75.80.+

\section{INTRODUCTION}

The ternary rare-earth intermetallic compound $\mathrm{TbNi}_{2} \mathrm{Ge}_{2}$ crystallizes in a body-centered tetragonal $\mathrm{ThCr}_{2} \mathrm{Si}_{2}$ structure and exhibits Ising-like antiferromagnetism with phase transitions into incommensurate and commensurate states at $\mathrm{T}_{\mathrm{N}}=16.7$ $\mathrm{K}$ and $\mathrm{T}_{\mathrm{t}}=9.6 \mathrm{~K}$ respectively; six additional metamagnetic phases have been observed in applied fields at $2 \mathrm{~K}$. The magnetic properties of $\mathrm{TbNi}_{2} \mathrm{Ge}_{2}$ are driven by indirect exchange interactions (presumably of the RKKY variety) between the magnetic rare-earth ions via the conductions electrons. $\mathrm{TbNi}_{2} \mathrm{Ge}_{2}$ has been studied by Bud'ko et al. as part of a more general inquiry into the magnetic behavior of rare-earth- $\mathrm{Ni}_{2} \mathrm{Ge}_{2}$ compounds [1]; references to earlier work may also be found in Ref. [1].

We have measured the linear thermal expansion and magnetostriction along the $c$-axis of $\mathrm{TbNi}_{2} \mathrm{Ge}_{2}$ (the axis along which the spins align) using a capacitive dilatometer constructed of OFHC copper and designed to operate in a Physical Property Measurement System available from Quantum Design Inc. A detailed description of the dilatometer will appear elsewhere [2].

\section{RESULTS AND DISCUSSION}

The linear thermal expansion of $\mathrm{TbNi}_{2} \mathrm{Ge}_{2}$ along its $c$-axis $(\alpha=\operatorname{d} \ln (\mathrm{L}) / \mathrm{dT}$ where $\mathrm{L}$ is the length of the sample, $0.85 \mathrm{~mm}$ here) is shown in Fig. 1. Features associated with the phase transitions at $T_{N}$ and $T_{t}$ are clearly visible. The broad maximum near $30 \mathrm{~K}$ is due to crystal field effects.

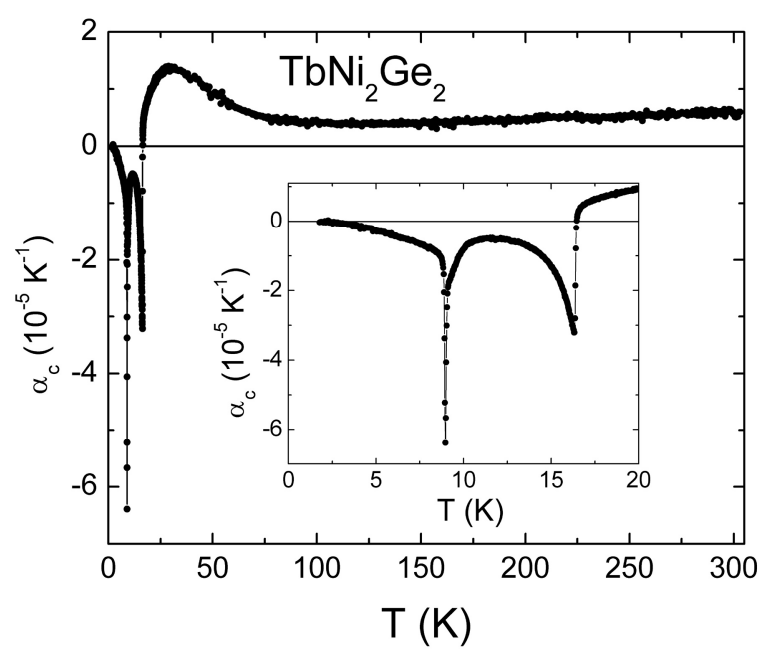

FIGURE 1. Linear thermal expansion of $\mathrm{TbNi}_{2} \mathrm{Ge}_{2}$ along its c-axis. The inset shows an expanded view of the low temperature phase transitions.

We first focus on the feature at $\mathrm{T}_{\mathrm{N}}$, the shape of which suggests that it is due to a $2^{\text {nd }}$ order phase transition. Combining the jump in thermal expansion $\left(\Delta \alpha=2.9 \times 10^{-5} \mathrm{~K}^{-1}\right)$ with the jump in molar specific heat from Ref. [1] $\left(\Delta C_{p}=-15 \mathrm{~J} / \mathrm{mol} \mathrm{K}\right)$, we can use the Ehrenfest relation 


$$
\frac{d T_{N}}{d P_{c}}=V_{M} T_{N} \frac{\Delta \alpha_{c}}{\Delta C_{p}},
$$

where $P_{c}$ is the uniaxial pressure along the c-axis and $\mathrm{V}_{\mathrm{M}}$ is the molar volume, to estimate the uniaxial pressure dependence of $\mathrm{T}_{\mathrm{N}}$ to be $-0.16 \mathrm{~K} / \mathrm{kbar}$.

The sharp feature at $T_{t}$ suggests that this phase transition is $1^{\text {st }}$ order, a suggestion that is supported by the presence of magnetic hysteresis across this phase boundary in higher magnetic fields [1]. However, the feature observed in $\mathrm{C}_{\mathrm{p}}$ at this temperature is relatively broad and therefore at odds with this suggestion. Should this be a $1^{\text {st }}$ order transition, a ClausiusClapyeron equation would apply:

$$
\frac{d T_{t}}{d P_{c}} \approx V_{M} \frac{\Delta(\Delta L / L)}{\Delta S},
$$

where $\Delta(\Delta \mathrm{L} / \mathrm{L})$ is the jump in the expansivity $\Delta \mathrm{L} / \mathrm{L}=$ $\mathrm{L}(\mathrm{T})-\mathrm{L}(0) / \mathrm{L}(0)$ at the phase transition $(\Delta \mathrm{L} / \mathrm{L}$ is determined by integrating $\alpha(\mathrm{T}) / \mathrm{T}$ across the transition), and $\Delta \mathrm{S}$ is the jump in molar entropy at the phase transition (determined by integrating $\mathrm{C}_{\mathrm{p}}(\mathrm{T}) / \mathrm{T}$ across the transition). Integrating our data shows that $\Delta(\Delta \mathrm{L} / \mathrm{L})$ is clearly finite at $\mathrm{T}_{\mathrm{t}}$, but integrating the specific heat data suggests that $\Delta \mathrm{S}$ is very close to zero. If this transition is $1^{\text {st }}$ order, Eqn. (1) suggests that the uniaxial pressure dependence of $T_{t}$ might be very large.

The magnetostriction of $\mathrm{TbNi}_{2} \mathrm{Ge}_{2}$ at $2 \mathrm{~K}$, measured along its $\mathrm{c}$-axis with magnetic fields applied parallel to the c-axis, is shown in Fig. 2 where $\Delta \mathrm{L} / \mathrm{L}=$ $\mathrm{L}(\mathrm{H})-\mathrm{L}(0) / \mathrm{L}(0)$. Magnetic hysteresis is observed in low fields along with a series of metamagnetic phase transitions consistent with the measurements of Bud'ko et al. [1] up to about $6 \mathrm{~T}$.

We observe an abrupt change in the slope of $\Delta \mathrm{L} / \mathrm{L}$ near $10 \mathrm{~T}$ at $2 \mathrm{~K}$ (marked by an arrow in Fig. 2, the derivative of the data with respect to magnetic field in the vicinity of $10 \mathrm{~T}$ is shown in the inset of Fig. 2). We suspect that this feature may signal a new fieldinduced phase transition although there is no corresponding signal of such a transition in high field magnetization measurements [1]. The feature was quite reproducible in our measurements and increased in field as the temperature increased (reaching $12.8 \mathrm{~T}$ at $30 \mathrm{~K}$; the feature was not observed at $50 \mathrm{~K}$ up to our maximum field of $14 \mathrm{~T}$ ). This temperature dependence suggests that the feature may be due to a spin-flop-like transition, though one would expect to see an associated metamagnetic-like feature in the field dependent magnetization, a feature that is not observed [1]. Understanding the origin and nature of this feature will require further study.

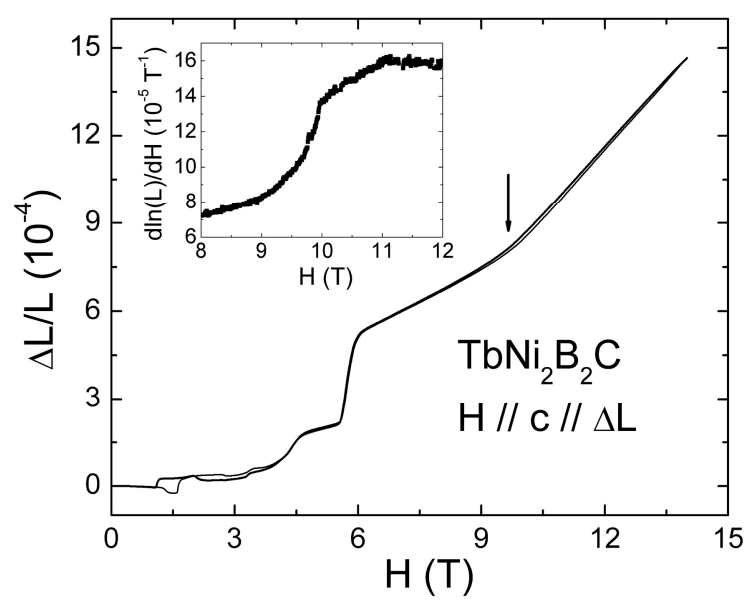

FIGURE 2. Magnetostriction of $\mathrm{TbNi}_{2} \mathrm{Ge}_{2}$ at $2 \mathrm{~K}$, measured along its c-axis in magnetic fields applied parallel to the caxis. The arrow denotes the position of a new feature. The inset shows a derivative of the data with respect to magnetic field in the vicinity of the feature.

\section{CONCLUSIONS}

We have measured the thermal expansion and magnetostriction of $\mathrm{TbNi}_{2} \mathrm{Ge}_{2}$ along its c-axis from room temperature to $2 \mathrm{~K}$ and in magnetic fields to 14 $\mathrm{T}$. Our results on the magnetic phase diagram agree with earlier results except for the observation of what may be a new field-induced phase above $10 \mathrm{~T}$ at low temperature. Application of thermodynamic relations to our results allowed us to estimate the affect of uniaxial pressure on the magnetic phase transitions in zero field.

\section{ACKNOWLEDGMENTS}

We are grateful to A.H. Lacerda and J.C. Cooley for several helpful discussions. Ames Laboratory is operated for the U.S. Department of Energy by Iowa State University under Contract No. W-7405-Eng-82. This work was supported by the Director of Energy Research, Office of Basic Energy Sciences and by the National Science Foundation under DMR-0305397.

\section{REFERENCES}

1. S.L. Bud'ko et al., J. Mag. Mag. Mater. 205, 53 (1999).

2. G.M. Schmiedeshoff et al. in preparation. 